\title{
Desenvolvimento e comparação de métodos para geração de séries temporais para imagens térmicas da tireoide
}

\author{
Wilian G. Fiirst ${ }^{1}$, Roger Resmini², Aura Conci ${ }^{3}$ \\ ${ }^{1}$ Instituto Federal de Mato Grosso Campus avançado Tangará da Serra (IFMT) \\ CEP - 78.300-000 - Tangará da Serra - MT - Brasil \\ ${ }^{2}$ Universidade Federal de Rondonópolis \\ CEP - 78.735-901, - Rondonópolis - MT - Brasil \\ ${ }^{3}$ Instituto de Computação \\ Universidade Federal Fluminense (UFF) - Niterói, RJ - Brasil \\ \{wilian.fiirstetga.ifmt.edu.br, rogerresminiegmail.com, \\ aconcieic.uff.br
}

\begin{abstract}
In order to analyze and possibly differentiate the pattern of carcinogenic regions of thyroid nodules, two methodologies to generate temporal series from region of interest points was proposed, discussed and compared. This methods are called Principal Axis orientation and Similarity Transformation Search Between Images. Simplified forms of these methods are also considered and implemented. Comparisons are done with both results, related their advantages and aspects. As far as we known there is no works employing the idea of the PA in this problem of following a same point through different images without brightness constancy.
\end{abstract}

Resumo. Para conseguir analisar e possivelmente diferenciar o comportamento de regiões da tireoide com nódulos cancerígenos, duas metodologias de construção de séries temporais dos pontos de uma determinada região de interesse foram propostas, discutidas e comparadas. Chamou-se esses métodos de orientação em relação ao Eixo Principal (Principal Axis - PA) e busca da transformação de Similitude (S) entre as imagens. Formas simplificadas destes métodos também são consideradas e implementadas. Até então o problema de seguir um mesmo ponto ao longo de diversas imagens é inexistente, cujas intensidades estejam variando, tenha sido considerado pelas metodologias propostas e em especial considerando a ideia do PA.

\section{Problema em estudo}

Descreve-se aqui um exemplo ilustrativo do problema. Nas Figuras 1 e 2, considerando que o voluntário seja inicialmente equivalente a um copo com água em uma temperatura de $37^{\circ}$ graus Celsius e o ambiente se encontra em uma temperatura de $28^{\circ}$ Celsius, suponha que as temperaturas estarão sendo avaliadas através da captura da cena por uma câmera, tal que essa consegue mostrar quanto os pontos variaram. 


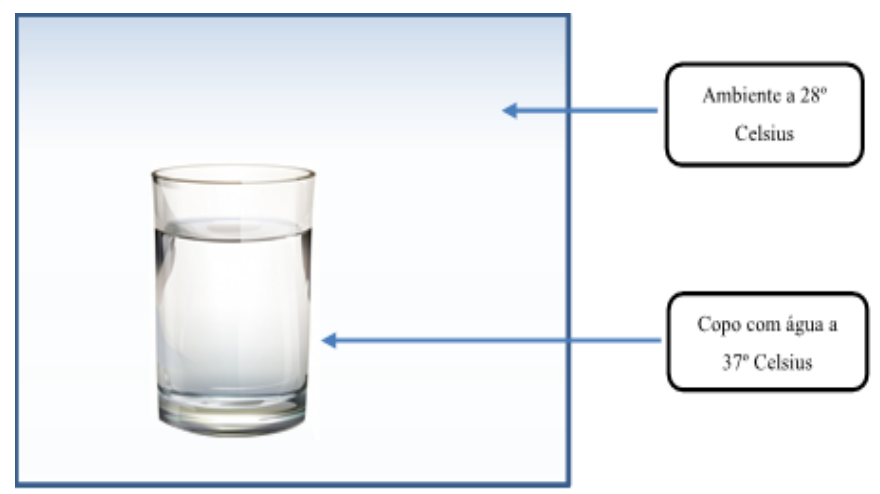

Figura 1 - llustrando o problema estudado

Inicia-se uma observação de 20 segundos e a cada segundo verifica-se como ficou um determinado ponto $\mathrm{G}$ (copo) e $\mathrm{H}$ (ambiente), então realiza-se hipoteticamente a seguinte anotação, $G=\{37 ; 36.9 ; 36.9 ; 36.8 ; 36.6 ; \ldots 35\}$ e $H=\{28 ; 28 ; 28 ; 28 ; \ldots 28\}$. Para facilitar como utilizar os dados destas séries, associa-se eles a sua ordem. Assim o primeiro será $G_{1}, H_{1}$, o segundo $G_{2}, H_{2}$ e um de ordem $J$ genérica será o elemento $J$ de qualquer um dos pontos $G_{J}, H_{J}$.

Agora imagine que ao invés de ser medida a temperatura, ela será capturada por uma câmera, que mede o quanto cada ponto de uma cena que ela captura emite, e, por um sistema (hardware e software) transforma a informação em temperatura. Após realização das capturas será possível construir uma série de imagens capturadas através da câmera.

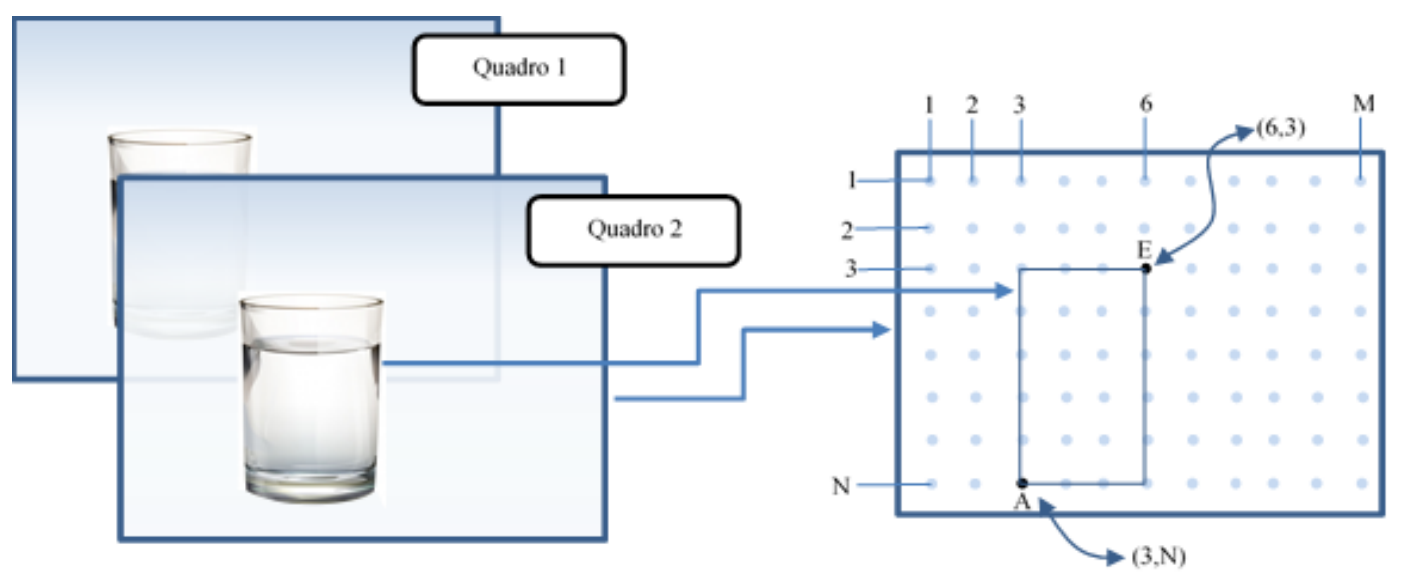

Figura 2 - Relação entre imagem e Termograma

Para cada ponto da temperatura da cena realiza-se a avaliação de temperatura do objeto correspondente. Assim em cada posição do array se tem um dado medido na câmera e que pode ser usado, mas se será associado ao copo ou ao ambiente, isso precisa ser definido. Para isso é "interessante usar os olhos", pois eles podem ajudar numa melhor identificação. Pode-se associar os dados da câmera à tons de cinza e com eles "pintar" uma região, fazendo uma associação adequada.

Para associar os tons de cinza ao array capturado, primeiro busca-se o valor mínimo e o valor máximo armazenado no array, associa-se esse mínimo a cor preta (tom de cinza 0) e o valor máximo à cor branca (tom de cinza 255) e usando uma transformação linear obtém-se a proporção de cinza de qualquer outro ponto do quadro $J$, que passa a 
ser reconhecido pela sua posição no array capturado e agora também por um tom de cinza.

Assim sendo, será usada essa associação entre cada ponto capturado e o tom de cinza, porque nesse quadro visível será possível identificar se um ponto $A$ ou $E$ pertence ao objeto ou não.

Agora imagine que entre uma captura e outra o copo "mudou" de lugar. Como o objetivo é construir uma série que permita ver como que as informações variaram ao longo do tempo, por exemplo, com que rapidez o copo esfriou, o mesmo ponto do copo deve ser medido a cada tempo, ou em outras palavras é importante garantir que um determinado ponto do objeto continue possível de ser seguido na construção da sequência. Mais concretamente, o problema em estudo é como sempre obter o conteúdo de um ponto na captura de um objeto que pode ter se movido, os pontos $A$ e $E$ da Figura 6 por exemplo. E para que esse problema seja resolvido esta pesquisa propõe dois métodos, um que chamou-se de Eixo Principal (Principal Axis - PA) e o outro chamou-se de Similitude S. As etapas que antecederam o principal objetivo dessa pesquisa influenciaram no bom alinhamento das imagens capturadas dos voluntários participantes para estudo dos pontos anatômicos nos termogramas. Visam a obtenção correta das imagens no Hospital Universitário Antônio Pedro utilizou-se um protocolo predefinido, refinou-se tal protocolo de captura, extraiu-se das matrizes de temperatura das imagens térmicas, gerando imagens em escala de cinza no processo de normalização das mesmas, segmentou-se as regiões de interesse usando limiarização e aplicou-se métodos que permitiram uma melhor extração da área de interesse. Tudo isso para atingir o objetivo principal que é seguir o mesmo ponto anatômico ao longo das imagens capturadas e propiciar uma correspondência adequada entre as posições das matrizes de temperatura.

\section{Metodologias propostas}

Rastrear a temperatura de um ponto anatômico do corpo do voluntário, em diferentes imagens térmicas da região de interesse, durante o exame para detecção de possíveis nódulos na tireoide é o problema a ser solucionado. Uma ideia seria aplicar uma metodologia para alinhamento de corpo rígido baseado na localização dos pontos das imagens a partir dos centroides e seus eixos principais de inércia, depois fazer as transformações entre imagens usando técnicas de análise tensorial. Essa ideia é a primeira linha de metodologia que denominou-se de método por Eixo Principal (Principal Axis PA).

Uma segunda linha de metodologia foi a utilização de técnicas tradicionais de registro adequadas as peculiaridades das imagens térmicas, como por exemplo as intensidades luminosas estarem variando entre as imagens a serem registradas. Todas as etapas dos métodos de registros baseados em transformações de similaridades entre pontos encontrados dentro da ROI, mas que não alterem o conteúdo destes pontos podem ser utilizadas nessa segunda linha.

A partir dessas metodologias, considerou-se também simplificações em cada uma delas para então obter transformações entre as imagens e posteriormente comparar os resultados em termos de tempo e de alguma função de qualidade que sejam adequadas à análise dos fatores que contribuem para verificação das vantagens e desvantagens de cada metodologia. 


\section{Resultados e comparações}

Os resultados foram comparados de três formas diferentes. Para a primeira forma de comparação dos métodos, escolheu-se 02 voluntários da base de imagens, e realizou-se comparações entre deslocamentos do pixel a ser seguido, assim como, mostrou-se as diferenças entre as séries encontradas entre ambas as técnicas completas (PA e S). Em uma segunda comparação, realizou-se a busca por um ponto anatômico em 12 voluntários da base de imagens, perfazendo um total de $10 \%$ dessa base, no intuito de encontrar as diferenças de deslocamentos nos eixos $x y$ e $P_{x y}$. Por último, comparou-se o método PA (usando apenas translação) e o método S (usando SIFT/RANSAC), aplicando correlações entre as imagens após uso dos métodos.

O computador utilizado para comparação dos resultados possui um processador Intel Core i7-3612QM 2.10GHZ, 4 Núcleos, 8 Threads, cache 6MB, além de 8 GB de memória RAM, disco de armazenamento de 1TB, sistema operacional Windows 7 Home Premium Service Pack 1.

Essa pesquisa propôs novas abordagens para o pré-processamento das imagens a serem usadas no exame térmico da tireoide. Esse pré-processamento visa especialmente como considerar o mesmo ponto anatômico ao longo de uma série de imagens termográficas extraídas da região da tireoide, capturadas durante o exame de um voluntário. Paralelamente também foi projetado e utilizado um suporte para apoio da cabeça, que é fixado na cadeira, em que o voluntário senta durante as aquisições de suas imagens. Esse ao ser usado pelo paciente fornece uma maior acomodação, reduzindo os deslocamentos que ocorrem no processo de captura. Também foi desenvolvido algoritmos para normalização das imagens e extração da região de interesse do pescoço, que serão utilizadas pelos especialistas para realização das segmentações manuais (que nesta linha de pesquisa servirão como ground truth nas etapas futuras).

Considerando que as imagens são objetos $2 \mathrm{D}$ e as propriedades física conhecidas de corpos em relação ao Centro de Massa ou Centro de Gravidade (CG) e Eixos Principais (PA), desenvolveu-se uma abordagem inovadora, em substituição ao registro tradicional de imagens. Também, uma outra metodologia de registro baseada em obtenção da transformação de Similitude para construção da transformação entre pares de imagens do mesmo voluntário foi desenvolvida. Além disso, para simplificar esses métodos, duas variações dos mesmos foram desenvolvidas, uma apenas considerando o $\mathrm{CG}$ e a outra aplicando os algoritmos SIFT e RANSAC para escolha automática dos pontos a serem usados no registro.

Para demonstrar a eficiência dos métodos propostos, comparações foram apresentadas. De acordo com os resultados obtidos, isto é, em comparação entre os métodos, aqueles que consideram um sistema de eixos passando pelo Centro de Gravidade (CG) ou os Eixos Principais (PA) se mostram mais eficientes, menos trabalhosos e mais corretos, pois uma vez que referenciados aos eixos fixos nos objetos representados na imagem, todos seus pontos passam a ser invariantes quando representados neste eixo, encontrando bons resultados para construção das séries temporais em um tempo muito menor e de forma totalmente automática.

Na aplicação dos métodos baseados em obtenção da transformação de Similitude, o esforço dispensado para criação das matrizes de Similitude depende muito de fatores visuais e de marcações manuais para criação dessas, deixando o processo mais oneroso. 
Na variação deste que considera usar algoritmos SIFT e RANSAC para substituir o trabalho manual das escolhas dos pontos, por outro lado, se mostrou instável nas imagens, pois as mesmas possuem poucas características constantes em todas as aquisições de um exame, essas características variaram seus valores de uma imagem para outra, inviabilizando parcialmente seu uso. Em um cômputo geral das comparações realizadas, o método $\mathrm{PA}$ foi sempre mais preciso e rápido em relação ao método $\mathrm{S}$, mesmo quando o método S utilizou-se de algoritmos para detecção de características.

\section{Comparações entre os métodos simplificados}

Para avaliar ambos os métodos na solução do problema proposto, resolveu-se comparar os métodos simplificados, usando uma derivação do método PA (apenas translação) e uma derivação do método $\mathrm{S}$ na forma automática (usando escolhas de pontos através dos algoritmos SIFT/RANSAC). Na avaliação dos resultados dos algoritmos aplicados, mediu-se o valor do coeficiente de correlações entre as ROI após a transformação de todos os 120 voluntários.

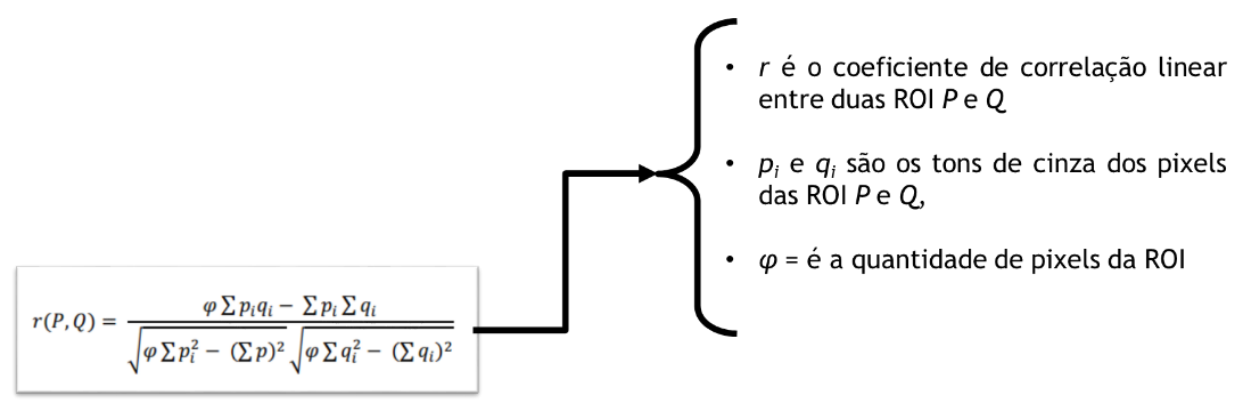

Figura 3 - Correlação aplicada

Sabemos que em um exame TID, mesmo sem nenhum movimento ou deformação do corpo, nunca haverá um coeficiente de correlação igual $+1,0$ devido as variações de tons de cinza sofridas pelos pixels das imagens no exame, até porque esta está associada ao estresse térmico induzido no corpo. Essa expressão $r$ é o coeficiente de correlação linear entre duas imagens $\mathrm{P}$ e Q, nesse caso entre a primeira imagem e as outras 19 imagens, que estão representadas por cada um de seus pixels da ROI. Assim pi e qi são os tons de cinza dos pixels das ROI P e Q, e fi = é a quantidade de pixels da ROI. Porém, para uma comparação entre os métodos simplificados, o seu uso se justifica, porque as imagens utilizadas sofrem a mesma variação de temperatura.
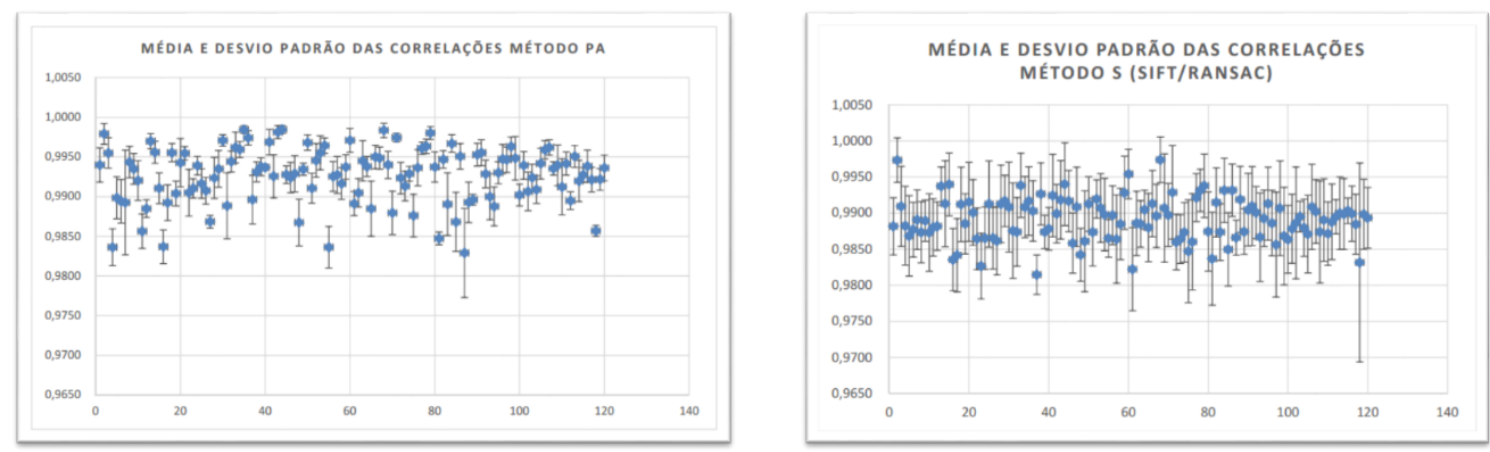

Figura 4 - Desvio Padrão dos métodos simplificados 
Ao analisar os valores obtidos por cada uma das técnicas na figura 4, observa-se melhores resultados com menores desvios padrões no método PA simplificado.

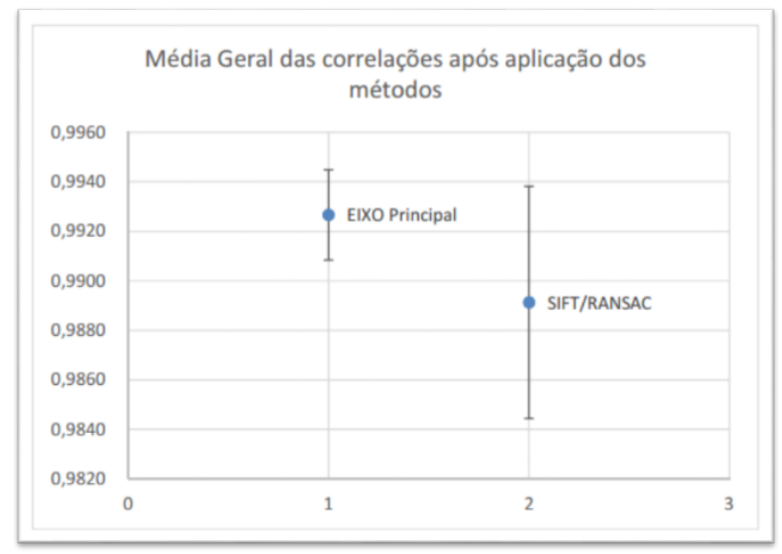

Figura 5 - Média Geral das correlações

Conforme figura 5, a média geral das correlações encontradas entre as imagens após transformação pelo método PA simplificado é de 0,9927 e no método $\mathrm{S}$ usando SIFT/RANSAC, atingiu-se uma média total de 0,9891.

As imagens utilizadas nesta dissertação podem ser encontradas no sitio virtual do laboratório de computação visual da UFF (http://visual.ic.uff.br), dentro do projeto de tireoides: http://visual.ic.uff.br/thyroid.

\section{Referências}

Borchartt, T. B.; Conci, A.; Lima, R. C. F.; Resmini, R.; Sanchez, A., (2013). Breast thermography from an image processing viewpoint: A survey. Signal Processing, Volume 93, pp. 2785-2803.

Brioschi, M. L.; Cimbalista, M.; Colman, D; Nakagawa, C. R.; Coltro, A.; Silveira, F., (2000). Benefícios da Imagem Digital Térmica Infravermelha no Diagnóstico dos Nódulos de Tireoide. Arquivos de Medicina, Volume Vol. 3, pp. 161-165.

Carvalho, G. A.; Graf, H.; Maciel, R. M. B.; Maciel, L. M. Z.; Maia, A. L.; Vaisman, M., (2013). Nódulo tireoidiano e câncer diferenciado de tireoide: atualização do consenso brasileiro. consenso em tireoide, pp. 240-264.

Conci, A., Azevedo, E. \& Leta, F. R., (2008). Computação Gráfica: Teoria e Prática. 2 ed. Rio de Janeiro: Campus/Elsevier.

Fiirst, W. G.; González, J. R.; Rosillo, E. C.; Souza, G. S. S.; Cicero, F. M.; Santos, E. V. V.; Vasconcelos, L. P.; Ribeiro, M. C.; Seixas, F. L.; Conci, A., (2017). On the use of tensor analysis for infrared image registration. niterói: Submetida ao IEEE ICC 2018 SAC Symposium E-Health Track.

Gonzalez, J. R.; Rodrigues, E. O.; Damiao, C. P.; Fontes, C. A. P.; Silva, A. C.; Paiva, A.; Li, H.; Du, C.; Conci, A., (2017). An Approach for Thyroid Nodule Analysis Using Infrared Images. BioEngineering; Book Title:Application of Infrared to Biomedical Sciences; $1^{a}$ edição. Ed. Springer Singapore, ISBN:978-981-10-3146-5, p. 451475. 